\title{
Design and analysis of plunger valve based on ansys
}

\author{
Gang Wang ${ }^{1,2}$, Rong Zhang ${ }^{1,2}$, a , Jing Wang ${ }^{1}$, Wei Wang ${ }^{1}$ and Maoyi Xie ${ }^{3}$ \\ ${ }^{1}$ Wuchang Institute of Technology, Wuhan, Hubei, China \\ ${ }^{2}$ Wuhan Polytechnic University, Wuhan, Hubei, China \\ ${ }^{3}$ Wuhan Yamei Valve Manufacturing Co, LTD, Wuhan, Hubei, China
}

\begin{abstract}
Water conservancy project generally has the characteristics of long running time and under high pressure and high flow rate and large flow, the director of the valve outlet exist cavitations and other issues, and the body vibration is more serious, the situation on the valve work. This paper based on the theory of fluid and the design of the piston flow adjusting valve, according to different work state of the fluid spray out to small columns or volute fan leaf form, at the exit to the intensity of the axial contraction and cancel each other out fluid impact, thus well control fluid state and eliminate the damage of the body fluid. The type of valve has been applied to the actual water conservancy project. Plunger Valve using SolidWorks modeling and static analysis, safety factor checking by means of stimulation, using fluid analysis software FLUENT was fluid simulation was used to simulate the fluid solid coupled analysis. So that the type of valve to meet the requirements of the work.
\end{abstract}

Keywords: plunger valve, cavitations, fluent, fluid solid coupling analysis.

\section{Introduction}

Valve is a kind of pressure pipe which is used to change the flow direction of the pipe section and the flow direction of the medium, to control the pressure, flow and temperature of the conveying medium [1]. In industrial pipelines, urban water supply system, transportation industry will be less piping accessories and equipment, the use of a large number of also indirectly promote nationalist economic development. Therefore, reasonable design of the valve of all walks of life is extremely important. This paper to install the Luohe Economic Development Zone, DN1000 type Plunger Valve, for example, in the process of water discharge, traditional valve equipment will due to the impact of the high speed flow and resulting in the edge of the body was badly damaged, especially the occurrence of cavitation. If the structure of the valve body is not reasonable design, it will lead to strong vibration of the valve in the working state; will cause the pipeline system as a whole is not stable. At the same time in the river or reservoir in the work of the valve will be due to the silt or impurities in the water resulting in obstruction of the valve body, so the design of the valve should be considered into the environmental factors. Plunger valve in this paper has good dissipation energy, pressure regulating and flow control, which would eliminate cavitations, no vibration, no noise, anti blocking ability in the pressure range and can achieve the water conservancy project expected effect.

a Corresponding author: meryer@sohu.com 


\section{Structure design of the plunger valve}

Plunger Valve, also known as the needle valve for water pipes, pumping station in the import and export pipeline and hydropower station water inlet pipe, mainly used the natural water flow, pressure regulation, also has reliable sealing and closing function[2]. Fluid through the deflector jet holes or fan-shaped leaves groove is throttled to multi beam flow, in the position of the axis of the valve body outlet collision, eliminating the impact energy of high speed flow; also because the collision occurred in the flow passage of the valve body cavity, the gas does not attached to the wall surface of the pipe rupture, so as to effectively reduce the influence of cavitations or erosion of the body, improve the service life of the valve. Drive shaft drives the crank and connecting rod movement and pushes the piston to move back and forth in the guide device. When the actuator drives the transmission shaft to rotate clockwise, a piston to move backwards, and open the valve; when the actuator to drive the stem counterclockwise rotation, the piston moves forward, close valve [3]. The main sealing ring is in the front of the flow deflector piston under the effect of the connecting rod, when being pushed through the main sealing ring to the fully closed position, before and after the valve pressure forced the main sealing ring clings to the outer wall of the sleeve to realize the truncation of the fluid.

\section{Sealing structure of plunger valve}

Plunger Valve comprises a valve body, a piston, a deflector, back cone inlet and a sealing ring is composed of main parts. Valve body is an integral structure design for cyclic axial symmetric flow channel, the cone in the lumen of the outlet end of the valve body, the valve body and the inner sleeve with rib support, in the middle of the piston is arranged in the inner sleeve, the crank and connecting rod driven piston reciprocating movement. There is a section between the inner sleeve and the guiding cone mouth, which is a section of open section without pipe wall, which is placed in the middle of a fan blade type or a squirrel cage type flow guiding device. Cone welded fan blade and the fluid which is energy dissipation at the axial line is diffused out from the wide angle of the inverted cone angle, which is beneficial for reducing the impact of the fluid on the river channel.

The double sealing method with the combination of $\mathrm{T}$ type anti extrusion inclined plane rubber strip soft seal and metal hard sealing is adopted, the sealing effect is good, and the bubble level seal and zero leakage can be realized. The rubber sealing ring is made of solid rubber with high hardness and $\mathrm{T}$ type, the slope can increase the effective sealing width, and the structure design of the $\mathrm{T}$ type rubber strip has the advantages of anti extrusion [4]. When the valve is closed, pressure, when the valve is opened (the throttle state) the main seal completely relaxed, not in contact with the throttle element, so no pressure and friction, so as to improve the life of the main seal, as shown in Figure 1.

The guide sliding seal of the piston and the inner sleeve adopts two special sealing rings, which can prevent the seal ring from curling and deforming, and improve the stability of the piston operation. Figure 2 shows. The special sealing ring adopts self sealing structure and has good sealing effect at high pressure, which effectively prevents the inner of the fluid from overflowing into the sleeve, and can automatically reduce the sealing pressure at low pressure, thereby effectively reducing the friction force.

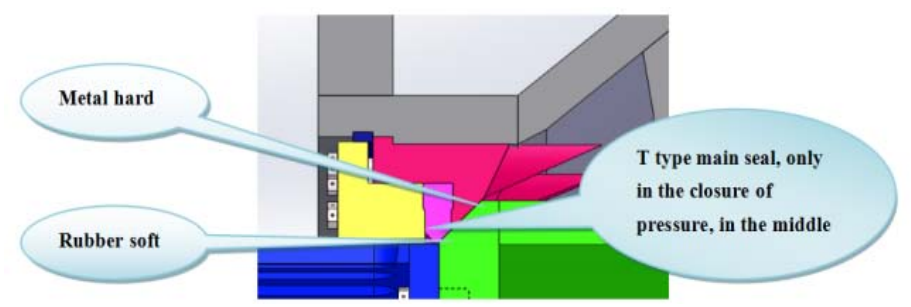

Figure1. Dual seal of soft and hard. 


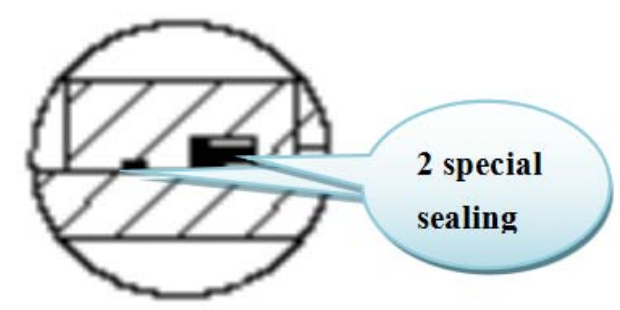

Figure 2. Inner seal.

\section{The fluid characteristics of plunger valve}

Design of the piston valve body into a whole, with high flow capacity, flow along the arc surface into the body, the valve body flow for axis symmetrical shape structure, ribbed plate is arranged between the valve body and the inner sleeve, the flow through the fluid does not produce turbulent [5]. Flow regulation is through a piston along the axial direction of the sleeve do linear motions to achieve, opening into a linear relationship with the flow, axial movement of the piston, change fluid flowing through the throttle width, to regulate the water flow, and flow with the valve open degree of linearity relationship [6]. Piston to move to any position, the valve cavity flow cross section are circular, the exit to the axis of contraction, attenuation and, therefore, does not cause pipeline vibration and cavitations broken ring, due to the passage of the axisymmetric structure, medium in high-speed flow, nor produced vibration [7].

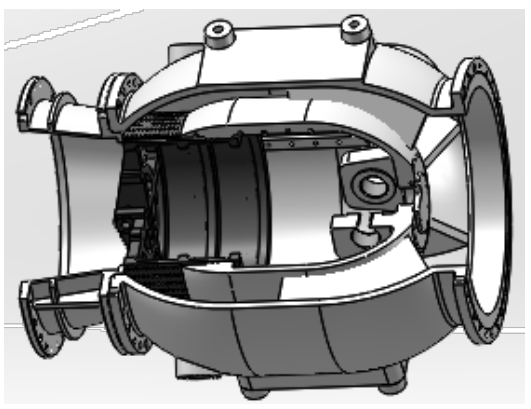

Figure 3. Closed mode.

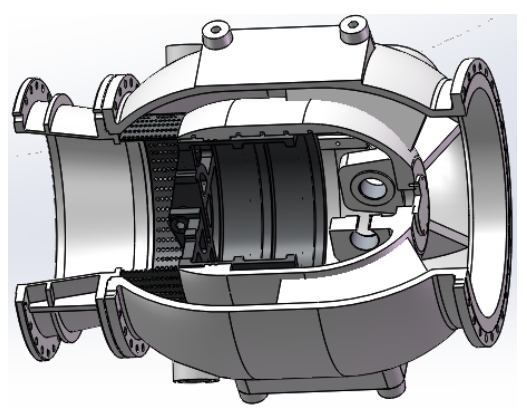

Figure 4. Fully open mode.

The guide is arranged in the sleeve, and the fluid passing through the valve body is contracted to the axis through the guide. Cylinder is a cylinder hole or fan-shaped groove in the front of the piston extends out a, section of the circumference, holes or grooves are symmetrically and uniformly distributed, when the medium flows through the holes or slots become high speed fluid, from the outside of the cylinder to cylinder pipe center jet impinging, so cavitations is defined in the center of the piston and the pipeline, not to valves and piping generated cavitations damage. 

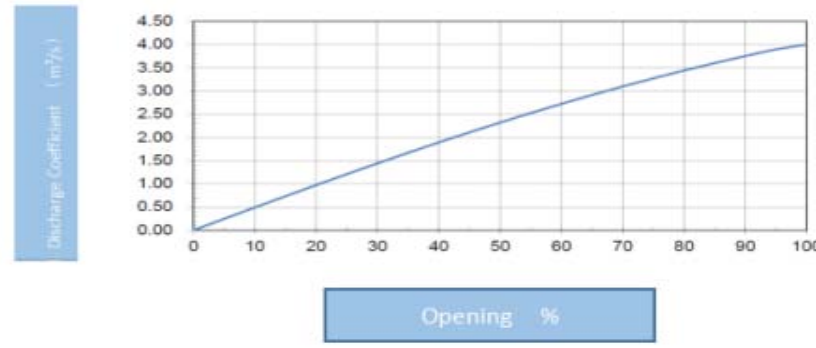

Figure 5. Flow characteristic curve.

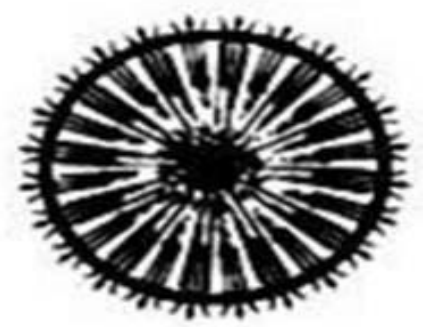

Figure 6. Rat cage guide.

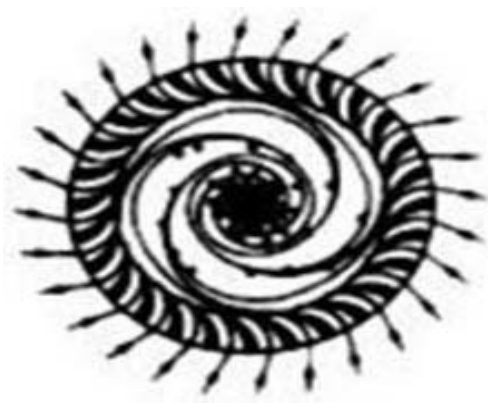

Figure 7. Fan vane.

\section{Parameters design of the plunger valve}

The design parameters of the valve according to the parameters according to the actual situation of the fluid, the components should be under the pressure of the medium in the pipeline. The valve body is designed to be cylindrical, and the outer end and the inner cavity of the outer valve body are oval shaped, and the middle part of the outer valve body and the inner sleeve are round. According to the classical fluid mechanics calculation the design principle of the method and flow adjusting valve, the valve adjust the position of the flow area, sleeve opening stroke and other parameters are determined, initially to determine the geometry and shape of flow channel, then use SolidWorks to establish entity model.

Plunger Valve, main technical performance pressure test standards in line with GB / T13927, shell test pressure of 1.5 PN, seal pressure test 1.1PN. Different pressure levels are according to customer's requirement. Several important design parameters as the following:

Cavitation coefficient. Valve design is mainly for the elimination of cavitations, improve the service life of the valve, while the smaller the gas, the more serious the phenomenon of cavitations. 


$$
\sigma=\frac{\mathrm{H}_{2}+\mathrm{H}}{\left(\mathrm{H}_{1}-\mathrm{H}_{2}\right)+\frac{\mathrm{V}}{2 \mathrm{~g}}}
$$

H1: Head height of the valve inlet, m; H2: Head height of the valve outlet, m;

$\mathrm{H}$ : Atmospheric pressure value, $\mathrm{m}$; V: Body flow rate, $\mathrm{m} / \mathrm{s}$;

g: Gravity acceleration, $9.8 \mathrm{~m} / \mathrm{s} 2$;

Nominal diameter. DN determined by the flow coefficient, access to the appropriate $\mathrm{Kv}$ characteristic curve to select appropriate DN, to determine the valve design to meet the state of fluid flow.

$$
\mathrm{K}_{\mathrm{v}}=\frac{10^{-3} \mathrm{q}_{m l}}{\sqrt{10\left(P_{1}-P_{2}\right) P_{L}}}
$$

$\mathrm{q}_{m l}$ : Flow through valve body mass, kg/h; P1: Valve before the pressure, MPa;

P2: Valve after the pressure, $\mathrm{MPa} ; \rho_{L}$ : Density of liquids, $\mathrm{kg} / \mathrm{m} 3$;

Wall thickness of the valve body. The valve body is a cylindrical design, and the design of the flange is connected with the pipe. The minimum wall thickness is based on thin wall cylinder, and is designed according to the fourth strength theory.

$$
\delta=\frac{P_{2} \times D N}{2 \times\left[\sigma_{L}\right] \times \eta-2 P_{2}(1-K)}+C
$$

$\mathrm{P} 2$ : Design pressure, $\mathrm{MPa}$; $\mathrm{DN}$ : Tube inside diameter , $\mathrm{mm} ; \mathrm{K}=0.6$;

$\left[\sigma_{\mathrm{L}}\right]$ : Allowable stress of materials, $\mathrm{N} / \mathrm{m} 2 ; \eta$ : Connection efficiency; $\mathrm{C}=6.3 \mathrm{~mm}$.

\section{Materials and technology}

Yield strength of the valve must meet the requirements. Parts shall be selected stainless steel and its corrosion resistance should be combined with the water quality requirements. Within a brass sleeve guide rail for supporting and guiding the manufacturing process using fine grinding, welding, in order to adapt to frequently adjust the action of the piston valve without wear. The piston cavity wall sleeve on the track, with accurate, reliable guide, mechanical piston movement will not slow or jam.

The valve sealing surface by laser cladding process, using high energy density of the laser will cover to the valve sealing surface of the nickel base alloy powder solution, forming a new kind of composite material and cladding layers of the grain is fine, compact structure, so it has high hardness, wear resistance and corrosion resistance also more excellent [8]. In the sealing surface to add a certain amount of carbide can significantly improve the wear resistance of the coatings [9].

Determines the quality of the sealing surface of the valve sealing performance. Generally using the method of grinding the valve sealing surface finishing, reducing and refining parts surface roughness, remove scratches, micro cracks and other surface defects. Sealing surface before the general should be fine grinding, after finishing the sealing surface can be directly grinding, the minimum grinding allowance is: the diameter of the margin is $8 \sim 20 \mu \mathrm{m}$; flat margin is $6 \sim 15 \mu \mathrm{m}$. The maximum value can be taken when the hand grinding or material hardness is higher, and the mechanical grinding or material hardness is lower [10].

\section{Finite element analysis}

Plunger Valve before the valve using Solidworks software modeling, using Fluent fluid analysis. Corresponding parameters, about electric Plunger Valve, DN1000, 1.5 MPa standard nominal 
pressure, are obtained and analyzed. The front valve body grid node number 497571, the unit number 284539 , the whole mesh quality is good, the base is fixed. By solving the operation, can get equivalent stress as shown in Figure 8a, Elastic strain equivalent as shown in Figure 8b, Total deformation as shown in Figure 8c.

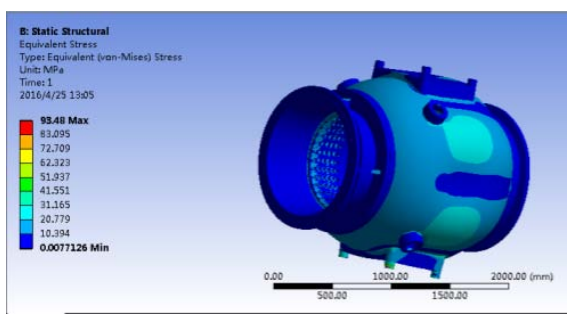

a. Equivalent stress map

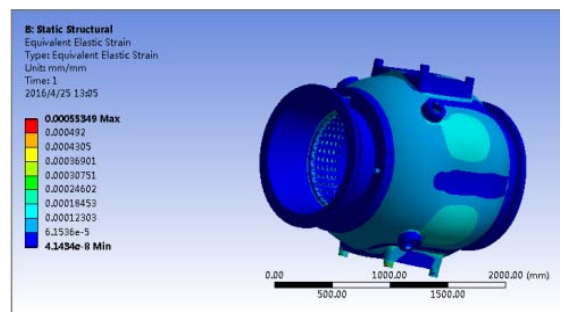

b. Strain distribution map

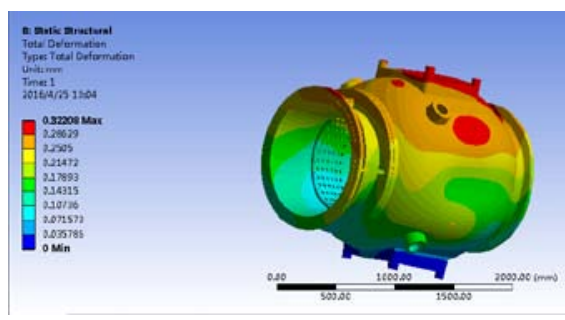

c. Displacement distribution map

Figure 8. Cloud map of the front body.

The front valve should stress, strain and displacement value as shown in Table 1, the fluent in $30 \mathrm{~m} 3 / \mathrm{s}$ velocity, the maximum von-mises is $93.48 \mathrm{MPa}$, less than the yield strength $310 \mathrm{MPa}$. Table safety factor 3.33162 is greater than 2 , design safety factor, so strength fully meet the requirements.

Table 1. Front body simulation results.

\begin{tabular}{l|l|l}
\hline \multicolumn{1}{c|}{ Type } & \multicolumn{1}{c}{ Minimum } & \multicolumn{1}{c}{ Maximum } \\
\hline Equivalent VON-mises stress $(\mathrm{MPa})$ & 0.0077126 & 93.48 \\
\hline Equivalent elastic strain $(\mathrm{mm})$ & $4.1434 \mathrm{e}-8$ & 0.00055349 \\
\hline Total deformation $(\mathrm{mm})$ & 0 & 0.32208 \\
\hline Minimum safety factor & \multicolumn{2}{|c}{3.3162} \\
\hline
\end{tabular}

The combined body of the front valve body, the guide cone mouth and the guide is introduced into the fluent:

1) In Geometry for fluid filling;Set the relevant parameters and mesh grid division;

2) To check the grid parameters, the grid volume is not negative, establish a Fluent analysis; Selection criteria model, the parameter set was proposed;

3) CFD-Post in the Results after the treatment, the flow rate of cloud images, as shown in Figure 9;

4) The operation result is introduced into Stress Static to carry on the fluid solid coupling analysis;

5) Material, mesh parameters selected, import fluent in solving the pressure value, concluded that the overall structure of the stress map, as shown in Figure 10. 


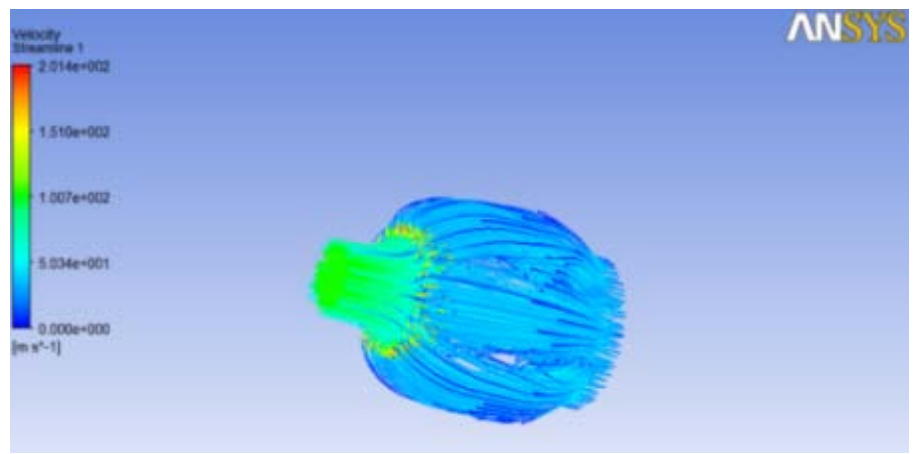

Figure 9. Fluid velocity cloud.

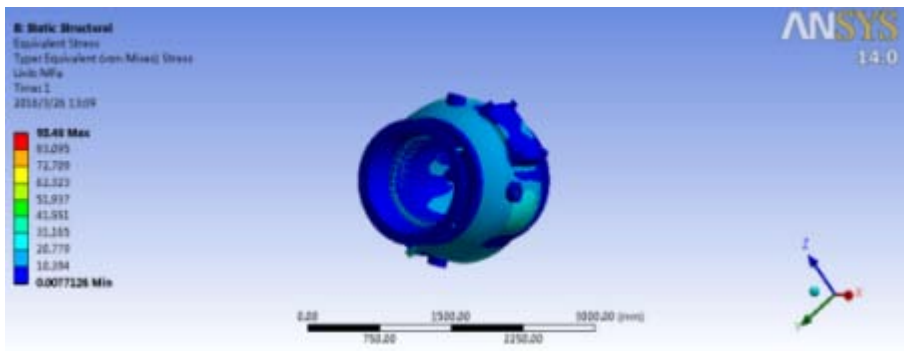

Figure 10. Global stress cloud.

The deflector and the guide conical oral combination in $30 \mathrm{~m}^{3} / \mathrm{s}$ speed should stress cloud chart shows that components of sufficient strength. Using FLUENT software to carry out the simulation , the valve and throttling of flow velocity, outlet valve of the flow can be accurately and visually displayed, numerical simulation results with the experimental data.The reliability of the model is verified in the turbulence model from the results.

\section{Control and driving system of plunger valve}

Plunger Valve with matic (integrated motor control unit), which is composed of two parts: actuators basic capacity of norm and installed in the actuator motor programming integration control unit matic. In the manual operation mode, the stroke switch and the executive should be set up. When the motor is in a stop state, the pull handle / automatic switch handle can be separated from the motor and the driving mechanism and the manual mechanism is connected with the transmission mechanism [11]. Control system and the surface of the high-quality anti-corrosion coating is at least 60 ion oxidation of polyurethane synthetic paint, can withstand mild atmospheric corrosion, suitable for outdoor installation [12].

\section{Conclusions}

Plunger Valvecan reduce cavitation damage in the structure, the entire valve, compared with other types of valves, the use of more long-life. The sealing system for the use of metal rubber double sealing form, so that it can greatly raise the working efficiency. The linear flow characteristics of the flow and the control flow is cut off well, let the control of the entire pipeline system is more convenient and agile. To sum up, and through the analysis of the fluid, both from the design principle or performance, piston type regulating valve can meet the engineering requirements.It can play a very important role in controlling the flow state of the fluid. 


\section{References}

1. Gong Yu Guilin Xu Yesong, Numerical simulation and flow field analysis of resistance coefficient of ball valve, Shaanxi Water Resources, 20-24 (2011)

2. water valve sample - Internet data - Baidu Library on http://www.baidu.com

3. Xue Ling, Application of pressure reducing valve in large drop pipe, Oil and Gas Storage and Transportation, 18, 22-25 (1999)

4. Piston type regulating valve product specifications -graphic on http://wenku.baidu.com

5. Liu Yanling; Chang Zhandong, Comparison and analysis of the structure and performance of the axial adjustment type control valve and the common control valve, Proceedings of the Sixth National Symposium on instrumentation and automation in petroleum and chemical industry, 18-20 (2007)

6. Xu Lei, The pressure regulating valve to reduce the loss rate and the rate of pipeline leakage pipe explosion using VAG piston flow, 34-37 (2008)

7. Xu Lei, New development of valve design, Assembly of the first working conference of the China Water Association and the exchange of experience in equipment management, 194-201 (2007)

8. The principle of laser cladding and laser processing technology, Chinese wiki.

9. Zhao Shihai, Jiang Xiuming, Wang Tiegang. Research status of alloy materials for wear resistant coating by laser cladding, 46-48 (2006)

10. Xu Liubin, On the development of valve grinding tool, Boiler manufacturing, 61-62 (2009)

11. Yang Shaoxuan Zhang Chen, Railway Transaction, 1996 first phase of the Research Institute of Zhengzhou Railway Bureau, Xi'an Railway Bureau, and Railway Administration.

12. VAG piston type regulating valve technical specification on http://wenku.baidu.com 